\title{
Nematofauna asociada a la rizosfera de papas (Solanum tuberosum) cultivadas en la zona productora del Cofre de Perote, Veracruz, México
}

\author{
Nematode fauna associated with the rhizosphere of potato crop (Solanum tuberosum) grown in \\ the region of Cofre de Perote, Veracruz, Mexico
}

\author{
Damaris Desgarennes ${ }^{1 *}$, Petra Sánchez-Nava ${ }^{1}$, Reyes Peña-Santiago ${ }^{2}$ y Gloria Carrión ${ }^{3}$ \\ ${ }^{1}$ Universidad Autónoma de Estado de México, Centro de Investigación en Recursos Bióticos, Facultad de Ciencias. Km. 14.5 Carretera Toluca- \\ Ixtlahuaca, Unidad San Cayetano de Morelos, 50000 Toluca, Estado de México, México. \\ ${ }^{2}$ Universidad de Jaén, Departamento de Biología Animal, Biología Vegetal y Ecología, Campus Las Lagunillas, Edificio B3, Dep. 146, Jaén, España \\ 23071. \\ ${ }^{3}$ Instituto de Ecología, A. C., Depto. de Biodiversidad y Sistemática. Km. 2.5 Carretera Antigua a Coatepec 351, Congregación El Haya, 91070, Xalapa, \\ Veracruz, México. \\ *Correspondencia: damaris.desgarennes@gmail.com
}

\begin{abstract}
Resumen. Se determinaron y clasificaron en grupos tróficos las especies de nematodos asociados a la rizosfera de Solanum tuberosum en suelos altamente infectados por el nematodo dorado de la papa (Globodera rostochiensis) en un ciclo de cultivo, en la zona productora del Cofre de Perote, Veracruz, México. Se identificaron 7 géneros (Aphelenchoides, Aphelenchus, Crassolabium, Mesodorylaimus, Plectus y Steinernema) y 8 especies (Aporcelaimellus obtusicaudatus, Ecumenicus monohystera, Acrobeles mariannae, Acrobeles singulus, Acrobeloides nanus, Cruznema tripartitum, Eucephalobus oxyuroides, y Globodera rostochiensis) en asociación con la rizosfera de papas cultivadas. Crassolabium sp. y E. monohystera se registran por primera vez para México.
\end{abstract}

Palabras clave: nematodos, cultivo de papa, grupos tróficos.

\begin{abstract}
Nematode species associated with the rhizosphere of Solanum tuberosum were identified and classified into trophic groups from soils highly infected by the golden potato cyst nematode (Globodera rostochiensis) in a single crop cycle in the producing zone on the Cofre de Perote, Veracruz, Mexico. Seven genera (Aphelenchoides, Aphelenchus, Crassolabium, Mesodorylaimus, Plectus and Steinernema) and 8 species (Aporcelaimellus obtusicaudatus, Ecumenicus monohystera, Acrobeles mariannae, Acrobeles singulus, Acrobeloides nanus, Cruznema tripartitum, Eucephalobus oxyuroides, and Globodera rostochiensis) were found in association with the rhizosphere of cultivated potatoes. Crassolabium sp. and E. monohystera are recorded for the first time in México.
\end{abstract}

Key words: nematodes, potato crop, trophic group.

Los nematodos son uno de los grupos más abundantes en el reino animal. Debido a su interacción con otros microorganismos tienen un papel importante en el suelo como reguladores en la descomposición y liberación de los nutrientes en los ecosistemas. No obstante, con excepción de los fitoparásitos, han sido poco estudiados tanto en suelos no perturbados como en aquellos dedicados a diversos cultivos (Ingham et al., 1985; Barker et al., 1994; Boag y Yeates, 1998). Se estima que existen aproximadamente un millón de especies de nematodos; de éstas, la mayoría (500 mil especies) son nematodos edáficos, de los que se conoce menos del 3\% (14 786 especies: 4105 nematodos fitoparásitos y 10684 de vida libre; Hammond, 1992; Hugot et al., 2001 y Esquivel, 2003).

Recibido: 13 septiembre 2008; aceptado: 17 febrero 2009
En México, el conocimiento de la nematofauna en suelos no perturbados y de cultivos es escaso y los estudios están enfocados principalmente a la detección y evaluación de la densidad de nematodos fitoparásitos (Montes-Belmont, 2002). Para el cultivo de papa, el cual ocupa el quinto lugar en producción en el país, sólo se han registrado nematodos que causan graves pérdidas económicas: Globodera rostochiensis (Wollenweber, 1923) Skarbilovich, 1959, Nacobbus aberrans (Thorne, 1935) Thorne y Allen 1944, y algunos fitoparásitos asociados: Aphelenchoides, Aphelenchus, Ditylenchus, Helicotylenchus, Hemicycliophora, Hoplolaimus, Meloidogyne, Pratylenchus, Tetylenchus, Tylenchorynchus, Tylenchus y Xiphinema (Manzanilla et al., 2002; MontesBelmont, 2002). El estudio del resto de la nematofauna presente en dicho cultivo y muchos otros, se ha dejado 
de lado a pesar de que pueden aportar conocimientos esenciales sobre el uso de nematodos como bioindicadores de las características del suelo, los efectos del cultivo, el estado de conservación del mismo y el manejo de las poblaciones de nematodos fitoparásitos (Barker et al., 1994; Bongers y Bongers, 1998; Yeates y Bongers, 1999; Yeates, 2003). En la región del Cofre de Perote, debido a la alta densidad de Globodera rostochiensis, los estudios relacionados con nematodos se han dirigido únicamente al monitoreo de la densidad y desarrollo de métodos de control para el nematodo dorado de la papa, sin prestar interés al resto de la nematofauna. Por lo tanto, el objetivo del presente estudio fue conocer las especies de nematodos asociadas a la rizosfera de papas cultivadas en la zona antes mencionada, perteneciente al estado de Veracruz, México.

Los nematodos se obtuvieron de 15 muestras de suelo provenientes de predios cultivados con papa y con alta densidad de Globodera rostochiensis (1 656 quistes/kg de suelo) (Núñez-Sánchez et al., 2003) en la localidad de Los Pescados, municipio de Perote en Veracruz, México [19 33' 41' 'N, 97 08' 53' 'O, 2980 m snm (INEGI, 2006)]. El clima de la zona es templado subhúmedo a semifrío $\mathrm{C}\left(\mathrm{W}_{2}{ }^{\prime}\right) \mathrm{B}$, con una temperatura media anual que oscila entre los 5 y $12^{\circ} \mathrm{C}$, con lluvias en verano que acumulan una precipitación anual promedio de $600 \mathrm{~mm}$ (Medina y Ángulo, 1990). Las muestras fueron tomadas siguiendo un patrón zig-zag (Haydock y Perry, 1998), a 5 cm del tallo de la planta de papa y $15 \mathrm{~cm}$ de profundidad y se transportaron al laboratorio en bolsas de plástico. Para la extracción de los nematodos, las muestras de suelo fueron procesadas por los métodos de tamizado-centrifugado (s'Jacob y van Bezooijen, 1984) y flotador de Fenwick (1940). Todos los ejemplares extraídos se fijaron en formol al $4 \%$ caliente y se transfirieron a glicerina anhidra (Seinhorst, 1959; 1962). Se examinaron 105 ejemplares montados de manera permanente en portaobjetos, para lo que se utilizó el método del anillo de parafina (s'Jacob y van Bezooijen, 1984). Los nematodos fueron determinados con ayuda de claves especializadas para cada orden: Siddiqi (2000) para Tylenchida; Hunt (1993) para Aphelenchida; Jairajpuri y Ahmad (1992) para Dorylaimida; Holovachov y De Ley (2006) para Plectida, y Andrássy (1984) para Rhabditida. Con base en la clasificación de hábitos alimenticios de los nematodos establecida por Yeates et al. (1993), los especímenes fueron colocados en los distintos grupos tróficos.

Se identificaron 7 géneros y 8 especies de nematodos pertenecientes a los órdenes Aphelenchida, Dorylaimida, Plectida, Rhabditida y Tylenchida (Cuadro 1); 2 taxa constituyen nuevos registros para México: Crassolabium sp. y Ecumenicus monohystera de Mann 1880; las medidas del único ejemplar encontrado de esta última especies son presentadas en el Cuadro 2.

Debido a que algunas de las especies pueden incluirse en 2 o más de los grupos tróficos en los que se les clasificó, en este estudio encontramos 7 especies bacteriófagas, 4 omnívoras, 2 principalmente micófagas y 2 fitoparásitas. Aphelenchoides sp. y Aphelenchus sp., que son micófagas, pueden ser también fitoparásitos al alimentarse de células epidérmicas o pelos radicales; inclusive, Aphelenchoides sp. se considera fitoparásito migratorio. Crassolabium sp., Mesodorylaimus sp., Ecumenicus monohystera y Aporcelaimellus obtusicaudatus (Bastiani, 1865; Altherr, 1968) son omnívoros; particularmente A. obtusicaudatus puede llegar a ser predador por ingesta o perforación de otros invertebrados como protozoos, rotíferos y enquitraeidos. Los fitoparásitos Paratylenchus sp. y $G$. rostochiensis son ectoparásito y parásito sedentario, respectivamente. Acrobeles mariannae Andrássy, 1968, Acrobeles singulus Heyns, 1969, Acrobeloides nanus (de Man, 1880) Anderson 1968, Cruznema tripartitum (von Linstow, 1906) Zullini, 1982, Eucephalobus oxyuroides (de Man, 1876) Steiner, 1936 y Plectus sp. se alimentan de bacterias. Steinernema sp., es el estadio infectivo de nematodos parásitos de insectos, además de bacteriófago en etapa juvenil.

Este estudio es el primer inventario en México de las especies de nematodos asociadas a la rizosfera de papas cultivadas. Sin embargo, los 15 taxa de nematodos encontrados en la rizosfera del cultivo de papa son pocos, comparados con los más de 30 géneros registrados por Yeates (1999) en suelos con vegetación no perturbada. En México, entre otros fitoparásitos, se detectaron Aphelenchus sp.y Aphelenchoides sp. en asociación con el cultivo de papa, pero sin causar daño a las plantas (MontesBelmont, 2002). Pen-Mouratov et al. (2008) registraron ambos géneros asociados con algunas leguminosas en el desierto de Tehuacán, en la región central de la República Mexicana. En otros países, Paratylenchus sp. se considera fitoparásito de ciruela (Prunus domestica L.) y apio (Apium graveolens L.); en México se ha registrado en asociación con leguminosas en el desierto de Chihuahua (Freckman y Virginia, 1985), pero no asociado al cultivo de papa con anterioridad. Desde la década de 1970, Globodera rostochiensis se ha registrado como uno de los fitoparásitos más importantes del cultivo de papa en México (Iverson, 1972). El resto de las especies que se encontraron en este estudio se consideran de vida libre y no se habían asociado al cultivo de papa en México.

Las diferencias entre las especies de nematodos en sistemas naturales y en agroecosistemas se refleja también en el número de grupos tróficos presentes en el suelo (Bongers y Bongers, 1998; Yeates, 2003). En este 
Cuadro 1. Géneros y especies de la nematofauna asociada a la rizosfera de cultivo de papa

\begin{tabular}{|c|c|c|c|c|}
\hline Orden & Familia & Género & Especie & Hábito alimenticio $* *$ \\
\hline \multirow[t]{2}{*}{ Aphelenchida } & Aphelenchoididae & Aphelenchoides & sp. & $\begin{array}{l}\text { Micófago; parásito migratorio; alimentación de } \\
\text { células epidérmicas y pelos radicales; alimentación } \\
\text { de algas, líquenes o musgos }\end{array}$ \\
\hline & Aphelenchidae & Aphelenchus & sp. & $\begin{array}{l}\text { Micófago; alimentación de células epidérmicas y } \\
\text { pelos radicales }\end{array}$ \\
\hline \multirow[t]{4}{*}{ Dorylaimida } & Aporcelaimidae & Aporcelaimellus & obtusicaudatus & Omnívoro; predador por ingesta o perforación \\
\hline & Dorylaimidae & Mesodorylaimus & $\mathrm{sp}$ & Omnívoro \\
\hline & Qudsianematidae & *Crassolabium & sp. & Omnívoro \\
\hline & & *Ecumenicus & monohystera & Omnívoro \\
\hline Plectida & Plectidae & Plectus & sp. & Bacteriófago \\
\hline \multirow[t]{6}{*}{ Rhabditida } & Cephalobidae & Acrobeles & mariannae & Bacteriófago \\
\hline & & Acrobeles & singulus & Bacteriófago \\
\hline & & Acrobeloides & nanus & Bacteriófago \\
\hline & & Eucephalobus & oxyuroides & Bacteriófago \\
\hline & Rhabditidae & Cruznema & tripartitum & Bacteriófago \\
\hline & Steinernematidae & Steinernema & sp. & $\begin{array}{l}\text { Bacteriófago, estado infectivo o de dispersión de } \\
\text { parásitos de animales }\end{array}$ \\
\hline \multirow[t]{2}{*}{ Tylenchida } & Heteroderidae & Globodera & rostochiensis & Parásito sedentario \\
\hline & Paratylenchidae & Paratylenchus & sp. & Ectoparásito \\
\hline
\end{tabular}

*Nuevo registro para México.

**Según Yeates et al., 1993

Cuadro 2. Caracteres morfométricos del ejemplar de Ecumenicus monohystera de Mann 1880 encontrado en la rizosfera de papas cultivadas

\begin{tabular}{lc}
\hline \multicolumn{2}{c}{ Índices y medidas } \\
\hline $\mathrm{n}$ & $1 \%$ \\
$\mathrm{~L}$ & $0.98 \mathrm{~mm}$ \\
$\mathrm{a}$ & 32.4 \\
$\mathrm{~b}$ & 4.51 \\
$\mathrm{c}$ & 34.14 \\
$\mathrm{c}$ & 1.51 \\
$\mathrm{~V}$ & 34 \\
Odontoestilete & $10.5 \mu \mathrm{m}$ \\
Largo faringe & $217.2 \mu \mathrm{m}$ \\
Largo expansión faríngea & $75 \mu \mathrm{m}$ \\
Diámetro a mitad del cuerpo & $30.24 \mu \mathrm{m}$ \\
Diámetro cuerpo a nivel anal & $19 \mu \mathrm{m}$ \\
Largo prerectum & $31 \mu \mathrm{m}$ \\
Largo cola & $28.7 \mu \mathrm{m}$ \\
\hline
\end{tabular}

inventario se encontraron 4 de los 8 grupos propuestos por Yeates et al. (1993): bacteriófagos, micófagos, omnívoros, fitoparásitos, así como el estadio infectivo de un parásito de animales. La ausencia de ingestores de sustrato, depredadores y consumidores de organismos eucariontes unicelulares, puede estar relacionada con las prácticas agrícolas de la zona de estudio, ya que los nematodos de dichos grupos tróficos generalmente se encuentran presentes en suelos sin alteraciones o con un bajo grado de perturbación (Bongers Bongers, 1998). Consideramos conveniente realizar el estudio de los nematodos de la rizosfera de las papas silvestres en las áreas boscosas del Cofre de Perote y contrastarla con la generada en nuestro estudio, para con ello obtener una mayor información sobre el equilibrio que las poblaciones de nematodos de vida libre guardan con los nematodos fitoparásitos.

Los autores agradecen al Consejo Nacional de Ciencia y Tecnología (CONACyT) por la beca otorgada al primer autor para realizar sus estudios de posgrado y la estancia de trabajo para la determinación de los especímenes en el Laboratorio de Nematología, Departamento de Biología Animal, Biología Vegetal y Ecología, Universidad de Jaén, España. Al Dr. Joaquín Abolafia por su apoyo en la determinación de los ejemplares del orden Rhabditida. El presente estudio fue financiado por el Instituto de Ecología, A. C. $(902-10 / 138)$. 


\section{Literatura citada}

Andrássy, I. 1984. Klasse Nematoda (Ordnungen Monhysterida, Desmocolecida, Araeolaimida, Chromadorida, Rhabditida). Gustav Fisher, Stuttgart. 509 p.

Barker, K. R., R. S. Hussey, L. R. Krusberg, G. W. Bird, R. A. Dunn, H. Ferris, V. R. Ferris, D. W. Freckman, C. J. Gabriel, P. S. Grewal, A. E. MacGuidwin, D. L. Riddle, P. A. Roberts y D. P. Schmitt. 1994. Plant and soil nematodes: societal impact and focus for the future. Journal of Nematology 26:127-137.

Boag, B. y G. W. Yeates. 1998. Soil Nematode Biodiversity in Terrestrial Ecosystems. Biodiversity and Conservation 7:617-630.

Bongers, T. y M. Bongers. 1998. Functional diversity of nematodes. Applied Soil Ecology 10:239-251.

Esquivel, A. 2003. Nematode fauna of Costa Rican protected areas. Nematropica 33:131-145.

Fenwick, D. W. 1940. Methods for recovery and counting of cysts of Heterodera shactii from. Journal of Helminthology 18:155-172.

Freckman, D. W. y R. A. Virginia. 1985. Plant-feeding nematodes in deep-rooting desert ecosystems. Ecology 70:1665-1678.

Hammond, P. M. 1992. Species inventory. In Global diversity status of the Earth's living resources, B. Groombridge (ed.). Chapman \& Hall, London. p. 17-39.

Haydock, P. P. y J. N. Perry. 1998. The principles and practice of sampling for the detection of potato cyst nematodes. In Potato cyst nematodes, biology, distribution and control, R. J. Marks y B. B. Brodie (eds.). CAB International, Wallingford, Oxfordshire. p. 61-74.

Holovachov, O. y P. De Ley. 2006. Order Plectida In Freshwater nematodes: ecology and taxonomy, E. Abebe, I. Andrássy y W. Traunspurger (eds.). CAB International, Wallingford, Oxfordshire. p. 611-647.

Hugot, J. P., P. Baujard y S. Morand. 2001. Biodiversity in helminths and nematodes as a field of study: an overview. Nematology 3:99-208.

Hunt, D. J. 1993. Aphelendhida, Longidoridae and Trichodoridae: Their systematics and bionomics. CAB International, Oxfordshire. $352 \mathrm{p}$.

INEGI (Instituto Nacional de Geografía, Estadística e Informática). 2006. Archivo histórico de localidades, Veracruz de Ignacio de la Llave. http://www.inegi.gob.mx/ geo/default.aspx?c=338\&e=30; consulta: 14.XI.2006.

Ingham, R. E., J. A. Trofymow, E. R. Ingham y D. C. Coleman. 1985. Interactions of bacteria, fungi, and their nematode grazers: effects on nutrient cycling and plant growth.
Ecological Monographs 55:119-140.

Iverson, L. G. K. 1972. Golden nematode-infestation found in Mexico. Plant Disease Reporter 49:281.

Jairajpuri, M. S. y W. Ahmad. 1992. Dorylaimida. Free-living, predaceus and plant-parasitic nematodes. E. J. Brill, Leiden. $458 \mathrm{p}$.

Manzanilla-López, R. H., M. A. Costilla, M. Doucet, J. Franco, R. N. Inserra, P. S. Lehman, I. Cid del Prado-Vera, R. M. Souza, and K. Evans. 2002. The genus Nacobbus Thorne \& Allen, 1944 (Nematoda: Pratylenchidae): Systematics, distribution, biology and management. Nematropica 32:149227.

Medina, A. y Angulo R. M. J. 1990. Atlas climático del municipio de Perote. Instituto de Ecología, Xalapa, Veracruz. 48 p.

Montes-Belmont, R. 2002. Nematología vegetal en México. Sociedad Mexicana de Fitopatología. Sonora, México. 98 p.

Núñez-Sánchez, A. E., M. C. Núñez-Camargo, G. Carrión y O. Rebolledo-Domínguez. 2003. Densidad de población de quistes de Globodera rostochiensis Woll. en el Cofre de Perote, Veracruz, México. Revista Mexicana de Fitopatología 21:207-213.

Pen-Mouratov, S., S. Rodríguez-Zaragoza y Y. Steinberg. 2008. The effect of Cercidium praecox and Prosopis laevigata on vertical distribution of soil free-living nematode communities in the Tehuacán Desert, México. Ecological Research 23:973-982.

Seinhorst, J. W. 1959. A rapid method for the transfer of nematodes from fixative to anhydrous glycerine. Nematologica: 4:67-69.

Seinhorst, J. W. 1962. On the killing, fixation and transferring to glycerin of nematodes. Nematologica 8:29-32.

Siddiqi, M. R. 2000. Tylenchida: parasites of plants and insects, 2a. edición. CAB International, Wallingford, Oxfordshire. $833 \mathrm{p}$.

s'Jacob, J. J. y J. van Bezooijen. 1984. A manual for practical work in nematology. Department of Nematology, Wageningen University. 77 p.

Yeates, G. W. 1999. Effects of plants on nematode community structure. Annual Review of Phytopathology 37:127-149.

Yeates, G. W. 2003. Nematodes as soil indicators: functional and biodiversity aspects. Biology and Fertility of Soils 37:199210.

Yeates, G. W. y T. Bongers. 1999. Nematode diversity in agroecosystems. Agriculture, Ecosystems and Environment 74:113-135.

Yeates, G. W., T. Bongers, R. G. M. de Goede, D. W. Freckman y S. S. Georgieva. 1993. Feeding habits in soil nematode families and genera-An outline for soil ecologists. Journal of Nematology 25:315-331. 